\title{
Current patent and clinical status of stimulator of interferon genes (STING) agonists for cancer immunotherapy
}

\author{
Mickaël Marloye1, Sean E Lawler² \& Gilles Berger ${ }^{*, 1,2(\mathbb{D})}$ \\ ${ }^{1}$ Microbiology, Bioorganic \& Macromolecular Chemistry Research Unit, Faculté de Pharmacie, Université libre de Bruxelles (ULB), \\ Boulevard du Triomphe, 1050 Brussels, Belgium \\ ${ }^{2}$ Harvey Cushing Neuro-Oncology Laboratories, Department of Neurosurgery, Brigham \& Women's Hospital, Harvard Medical \\ School, Boston, MA 02115, USA \\ *Author for correspondence: gpberger@bwh.harvard.edu
}

"The combination of STING agonists with current immunotherapy regimens is showing great promise, considering that a cold tumor microenvironment appears to be the Achilles heel of immunotherapy"

First draft submitted: 10 June 2019; Accepted for publication: 13 June 2019; Published online: 29 July 2019

Keywords: cancer immunotherapy • cyclic dinucleotides $\bullet$ immune response $\bullet$ STING $\bullet$ type I interferon

Immunotherapy has in recent years shown its potential to transform the treatment of cancer through triggering antitumor $\mathrm{T}$ cell responses via immune checkpoint blockade. This approach has led to outstanding responses in previously untreatable tumors. Stimulator of interferon genes (STING) has now been revealed as a key player in the field and a critical mediator of innate immune-sensing of cancer, and in turn, as a promising target for immunotherapy. Pharmacological activation of the host STING pathway can trigger T cell-mediated tumor regression and make immunologically 'cold' tumors 'hot'. This can help to overcome local immunosuppressive environments as seen in cancer, and can increase the effectiveness of classical immune checkpoint blockade.

Immune evasion is now well established as a major hallmark of cancer [1]. Reactivation of the immune system and redirection of the immune response toward the tumor has been a major focus of attention in the past few years and immunotherapy has earned its place in the anticancer therapeutic arsenal, alongside the traditional pillars of chemotherapy, radiotherapy and surgery. The remarkable impact of immunotherapy on previously untreatable tumors and advanced metastatic cancers led the Nobel prize in Medicine or Physiology in 2018 to be awarded to James Allison and Tasuku Honjo for their discovery of immune checkpoint blockade. This approach consists of releasing the local inhibition of the immune response within the tumor microenvironment, using monoclonal antibodies directed against the cytotoxic T lymphocyte antigen 4 (CTLA-4) [2] and the programmed death 1 (PD-1) surface receptors [3], or its ligand, PD-L1 [4]. Despite impressive clinical outcomes and more than 50 Phase III trials involving anti-CTLA4, anti-PD1 and anti-PD-L1 monoclonal antibodies [5], responses are highly variable between patients and tumor types, with anti-CTLA4 therapy inducing positive response in approximately only $15 \%$ of patients with metastatic melanoma [6].

\section{The cGMP-AMP synthase (cGAS)-STING-IRF3 pathway, a novel target for immunotherapy}

Tumor infiltration by $\mathrm{T}$ cells has been shown to be a prerequisite for an efficient response to immunotherapeutic treatments [7]. 'Cold', nonimmunogenic tumors are less responsive to immune checkpoint inhibitors; therefore, promoting $\mathrm{T}$ cells infiltration in the tumor microenvironment is a promising approach to increase efficacy of immunotherapeutic drugs and 'heating' up the tumors. The endoplasmic reticulum adaptor protein stimulator of interferon genes (STING, gene ID TMEM173) activates innate immune responses through the production of type I interferon. Cytoplasmic DNA, which can be either pathogen or tumor-derived, triggers the activation of the host cGAS-STING-IRF3 pathway, mediated by the cGMP-AMP synthase (cGAS), a cytosolic enzyme that generates 2',3'-cGAMP (cyclic Guanosine monophosphate [GMP]-Adenosine monophosphate [AMP]) upon binding to 
double-stranded DNA. This endogenous cyclic dinucleotide (CDN) then binds to STING and promotes the induction of the immune cascade [8]. This leads to activation and infiltration of $\mathrm{T}$ cells and promotes antitumor response [9]. The development of STING agonists has rapidly grown as a novel class of immunotherapy, either alone or in combination with immune checkpoint blockade. STING agonists can be grouped in two main subtypes: nucleotidic or non-nucleotidic agonists.

\section{Nucleotidic STING agonists}

The prokaryotic c-di[GMP] and eukaryotic 2',3'-cGAMP CDNs have demonstrated promising antitumor activity and potent immunostimulatory effects both in vitro and in vivo [10,11]. These natural CDNs are however poor drug candidates for anticancer therapies, owing to their instability and high polarity, which limits their diffusion across cell membranes. This led to the development of synthetic CDNs. The 'dithio' analog, ADU-S100 (also called MLRR-S2-CDA or MIW815) [12], uses sulfur-modified phosphodiester linkages on a c-di[AMP] scaffold to increase its resistance to enzymatic degradation [13]. Since 2012, the preparation and utilization of ADU-S100 has been patented by Aduro Biotech (CA, USA) [14-20]. Encouraging preclinical results have brought ADU-S100 forward to Phase I clinical trials, where it is administered by intratumoral injection to patients with advanced metastatic solid tumors or lymphomas, alone or in combination with ipilimumab (NCT02675439) or with an anti-PD1 (NCT03172936). Partial results have been disclosed and are encouraging but more complete data will be available in 2020. Other novel synthetic CDNs have been patented by Merck (NJ, USA) [21,22]. One of which, MK-1454, is currently undergoing Phase I trial for mono- and combination therapy with pembrolizumab for the treatment of solid tumors and lymphoma (NCT03010176), and the first results are expected in 2021. GlaxoSmithKline (London, UK) developed and patented synthetic CDNs in 2015 [23], including fluorinated derivatives as STING modulators. More recently, Bristol-Myers Squibb (NJ, USA) filed a patent on novel CDNs as anticancer agents [24].

\section{Non-nucleotidic STING agonists}

5,6-dimethylxanthenone-4-acetic acid (DMXAA), also known as vadimezan or ASA404, was first discovered as a vascular disrupting agent [25], but is now recognized as a non-nucleotidic STING agonist and has rapidly entered clinical trials. However, DMXAA is a poor agonist of human STING and ultimately failed in Phase III clinical trials in combination with paclitaxel and carboplatin for the treatment of non-small cell lung cancer [26]. Yet various combinations of DMXAA with marketed anticancer drugs have been patented, including platinum compounds, vinca alkaloids, alkylating agents, anthracyclines, topoisomerase I inhibitors, antimetabolites and topoisomerase II inhibitors $[27,28]$. Combinations with EGFR signaling inhibitors were also patented [29], including Erbitux ${ }^{\mathrm{TM}}$ (cetuximab), a monoclonal antibody binding to EGFR, Tarceva ${ }^{T M}$ (erlotinib) and Iressa ${ }^{T M}$ (gefitinib), which are small molecule EGFR inhibitors. A European patent was filed for DMXAA combination with gemcitabine, 5fluorouracil, doxorubicin and irinotecan [30]. DMXAA further served as a proof-of-concept for designing novel non-nucleotidic STING agonists but without significant success, although one group tried to overcome the low affinity of the DMXAA scaffold for human STING by adding a hydrogen bond donor on the C7 position [31,32]. Currently, no non-nucleotidic STING agonists have reached clinical trials but GSK have disclosed two novel amidobenzimidazole STING agonists inducing significant tumor growth inhibition using in vivo mice tumor models [33] and have filed their discovery under the patent WO2019069270A [34].

\section{Perspective}

Large pharmaceutical companies have dominated the development of CDNs as STING agonists, but there is still room for the development of more drug-like non-CDN structures. There is no doubt that this emerging and exciting field will continue to garner much attention from the scientific community. Rational drug design and modern computational approaches are expected to provide more insights as the crystal structure of human STING was solved in 2013 [35], additionally the binding modes of active ligands have been comprehensively studied [31,33,36].

The combination of STING agonists with current immunotherapy regimens is showing great promise, considering that a cold tumor microenvironment appears to be the Achilles heel of immunotherapy, and that STING pathway activation could 'heat up' the tumor and trigger infiltration by immune cells, which in turn can be unleashed by the checkpoint blockade. This would allow the ability to target tumors refractory to immune checkpoint blockade with a low risk of systemic side effects, and increase the likelihood of STING agonists to become important cancer drugs in the near future. 


\section{Financial \& competing interests disclosure}

The authors have no relevant affiliations or financial involvement with any organization or entity with a financial interest in or financial conflict with the subject matter or materials discussed in the manuscript. This includes employment, consultancies, honoraria, stock ownership or options, expert testimony, grants or patents received or pending, or royalties.

No writing assistance was utilized in the production of this manuscript.

\section{References}

1. Hanahan D, Weinberg RA. Hallmarks of cancer: the next generation. Cell 144(5), 646-674 (2011).

2. Leach DR, Krummel MF, Allison JP. Enhancement of antitumor immunity by CTLA-4 Blockade. Science 271(5256), 1734-1736 (1996).

3. Ishida Y, Agata Y, Shibahara K, Honjo T. Induced expression of PD-1, a novel member of the immunoglobulin gene superfamily, upon programmed cell death. EMBO J. 11(11), 3887-3895 (1992).

4. Dong H, Zhu G, Tamada K, Chen L. B7-H1, a third member of the B7 family, co-stimulates T-cell proliferation and interleukin-10 secretion. Nat. Med. 5(12), 1365-1369 (1999).

5. Zhu H-F, Li Y. Small-molecule targets in tumor immunotherapy. Nat. Prod. Bioprospect. 8(4), $297-301$ (2018).

6. Ribas A, Wolchok JD. Cancer immunotherapy using checkpoint blockade. Science 359(6382), 1350-1355 (2018).

7. Gajewski TF, Schreiber H, Fu Y-X. Innate and adaptive immune cells in the tumor microenvironment. Nat. Immunol. 14(10), 1014-1022 (2013).

8. Sun L, Wu J, Du F, Chen X, Chen ZJ. Cyclic GMP-AMP synthase is a cytosolic DNA sensor that activates the type i interferon pathway. Science 339(6121), 786-791 (2013).

9. Corrales L, Gajewski TF. Molecular pathways: targeting the stimulator of interferon genes (STING) in the immunotherapy of cancer. Clin. Cancer Res. 21(21), 4774-4779 (2015).

10. Ohkuri T, Kosaka A, Ishibashi K et al. Intratumoral administration of cGAMP transiently accumulates potent macrophages for anti-tumor immunity at a mouse tumor site. Cancer Immunol. Immunother. 66(6), 705-716 (2017).

11. Deng L, Liang H, Xu M et al. STING-dependent cytosolic DNA sensing promotes radiation-induced type I interferon-dependent antitumor immunity in immunogenic tumors. Immunity 41 (5), 543-852 (2014).

12. Corrales L, Glickman LH, McWhirter SM et al. Direct activation of STING in the tumor microenvironment leads to potent and systemic tumor regression and immunity. Cell Rep. 11(7), 1018-1030 (2015).

13. Li L, Yin Q, Kuss P et al. Hydrolysis of 2'3'-cGAMP by ENPP1 and design of nonhydrolyzable analogs. Nat. Chem. Biol. 10(12), 1043-1048 (2014).

14. Aduro Biotech, Inc.: WO2013185052A1 (2013).

15. Aduro Biotech, Inc.: US9695212B2 (2017).

16. Aduro Biotech, Inc.: US20170218008A1 (2017).

17. Aduro Biotech, Inc.: US9724408B2 (2019).

18. University of Chicago and Aduro Biotech, Inc.: EP3071209A1 (2016).

19. University of Chicago and Aduro Biotech, Inc.: EP3071209A4 (2017).

20. Aduro Biotech, Inc.: AU2013358892B2 (2018).

21. Merck Sharp \& Dohme Corp.: WO2017027645A1 (2017).

22. Merck Sharp \& Dohme Corp.: CA2995365A (2017).

23. Glaxosmithkline Intellectual Property Development Ltd.: WO2015185565A1 (2015).

24. Bristol-Myers Squibb, Co.: WO2019079261A1 (2019).

25. Bibby MC, Phillips RM, Double JA, Pratesi G. Anti-tumour activity of flavone acetic acid (NSC 347512) in mice - influence of immune status. Br. J. Cancer 63(1), 57-62 (1991).

26. Lara PN, Douillard JY, Nakagawa K et al. Randomized Phase III placebo-controlled trial of carboplatin and paclitaxel with or without the vascular disrupting agent vadimezan (ASA404) in advanced non-small-cell lung cancer. J. Clin. Oncol. 29(22), 2965-2971 (2011).

27. Cancer Research Technology Ltd.: EP1759694A2 (2007).

28. Cancer Research Technology Ltd.: EP1423105B1 (2008).

29. Antisoma Plc.: WO2007023307A1 (2007).

30. Cancer Research Technology Ltd.: EP1423105B9 (2009).

31. Gao P, Zillinger T, Wang W et al. Binding-pocket and lid-region substitutions render human STING sensitive to the species-specific drug DMXAA. Cell Rep. 8(6), 1668-1676 (2014). 
32. Hwang J, Kang T, Lee J, Choi B-S, Han S. Design, synthesis, and biological evaluation of C7-functionalized DMXAA derivatives as potential human-STING agonists. Org. Biomol.

Chem. (2018). https://pubs.rsc.org/en/content/articlelanding/2019/OB/C8OB01798K\#!divAbstract

33. Ramanjulu JM, Pesiridis GS, Yang J et al. Design of amidobenzimidazole STING receptor agonists with systemic activity. Nature 564(7736), 439-443 (2018).

34. Glaxosmithkline Intellectual Property Development Ltd. WO2019069270A (2019).

35. Gao P, Ascano M, Zillinger T et al. Structure-function analysis of STING activation by c $\left[\mathrm{G}\left(2^{\prime}, 5^{\prime}\right) \mathrm{pA}\left(3^{\prime}, 5^{\prime}\right) \mathrm{p}\right]$ and targeting by antiviral DMXAA. Cell 154(4), 748-762 (2013).

36. Shang G, Zhang C, Chen ZJ, Bai XC, Zhang X. Cryo-EM structures of STING reveal its mechanism of activation by cyclic GMP-AMP. Nature 567(7748), 389-393 (2019). 